\title{
Triangular potential effects on the fermi velocity renormalization in 8-Pmmn borophene
}

\author{
Defne Akay*
}

Ankara University, Faculty of Science, Department of Physics, 06100 Tandoğan, Ankara, Turkey, ORCID ID orcid.org/ 0000-0001-8990-007X

\section{ARTICLE INFO}

\section{Article history:}

Received 01 August 2019

Revised form 09 September 2019

Accepted 20 September 2019

Online 30 September 2019

Research Article

DOI: 10.30728/boron.599559

\section{Keywords:}

8-Pmmn Borophene,

Fermi velocity,

Triangular potential.

\begin{abstract}
A novel mechanism has been reported for the intrinsic electronic properties of the 8-Pmmn borophene to investigate the electronic energy spectrum and spectrum parameters of the structure. To examine the structure, we extract the energy spectrum of the Hamiltonian for the monolayer borophene by using analytical method. Additionally, for the intrinsic properties, first we obtained the bare Fermi velocity which findings of the study are consistent with experimental results. After obtained the bare state Fermi velocity 8-Pmmn borophene, dressed with triangular potential and renormalized Fermi velocity has been obtained. Corresponding renormalized Fermi velocity reshaped with the triangular potential which has non-negligible contribution to the intrinsic properties of borophene. This finding has important implications for developing electronic devices which made from the boron due to increasing the controllability of the structure.
\end{abstract}

\section{Introduction}

The first 2-dimensional material was isolated from graphene in 2004 [1] by Andre Geim and Konstantin Novoselov at the University of Manchester. The scientists received the 2010 Nobel Prize in physics. Since the discovery of the graphene, further researches have been realized on the other 2-dimensional materials because of their extraordinary electronic, optical and chemical properties [2-9]. Main application areas of the 2-dimensional materials are biological engineering, electronics, sensors, optoelectronics, composite materials, medicine, photovoltaic systems and energy storage [10-14]. With these applications great advances have been achieved, nevertheless none of these 2D materials has been gained attention for commercial applications, except of graphene. An international team of scientists led by N.P. Guisinger at Argonne National Laboratory; A. R. Oganov at Stony Brook University; M. C. Hersham at Northwestern University and Moscow Institute of Physics together with Skolkovo Institute of Science and Technology has been realized a large amount of research and synthesized borophene [15]. Borophene and graphene have many similarity due to their single atomic thickness sheets and they have great strong structure which display an extremely flexibility. However, they have substantial distinction on their lattice structures. Borophene is a crystalline allotrope of boron which has non-metallic nature as a bulk structure. Borophene is a highly anisotropic material which exhibit semiconducting and metallic behavior. Very recently, the 2-dimensional promising nanostructure has been predicted theoretically and effects of particle-hole symmetry breaking have been reported by optical conductivity [16]. In addition, abinitio properties of 8-Pmmn borophene has been studied, recently [17] and Li et al.[18] identified the energy spectrum of the 8-Pmmn borophene and showed it is ideal material to analyze Klein tunneling in the presence of anisotropy. Morover, similar to the graphene magnetic field effects has also been addressed in this material [19] but it still needs many work to specify its practical applications. Borophene has been started to frequently seen in the literature, but to date there is no study about triangular potential effect on the Fermi velocity. The research may be defined as a systematic process which consists of three elements or components: (1) electronic energy spectrum is anisotropic (2) the anisotropic spectrum extended with triangular potential, and (3) analysis and interpretation of triangular potential on the Dirac Fermion velocity which has two direction $v_{x}$ and $v_{y}$ are velocities along the $x$ and $y$ coordinates, respectively.

\section{Theoretical model}

Single-particle low-energy effective continuum model of the anisotropic Dirac model which corresponds to 8-Pmmn borophene with triangular potential as,

$\widehat{H}_{\rho}=\rho\left(v_{x} \hat{P}_{x} \sigma_{x}+v_{y} \hat{P}_{y} \sigma_{y}+v_{t} \hat{P}_{y} \sigma_{0}\right)$

Where the first two terms correspond to kinetic energy 
term and the third term described the tilted nature of the Dirac cones. $\rho$ represents to valley index and $+(-)$ corresponds to $K\left(K^{\prime}\right)$ Dirac points. The $v_{x}$ and $v_{y}$ are velocities along the $x$ and $y$ coordinates, respectively. They are given by $\left\{v_{x}, v_{y}\right\}=\left\{0.86 v_{F}, 0.69 v_{F}\right\}$ in units of $v_{F}=10^{6} \mathrm{~m} / \mathrm{s} . v_{t}$ is anisotropic velocity arises due to tilted Dirac Fermions and its values is $0.32 v_{F} . \sigma_{0}$ is the identity matrix and $\sigma_{i}$ are denotes to Pauli matrices. $\hat{P}_{x}$ and $\hat{P}_{y}$ are the momentum operators. Corresponding energy dispersion of the Hamiltonian can be obtained as,

$E_{\rho}^{\lambda}=\rho \hbar v_{t} k_{y}+\lambda \hbar \sqrt{v_{x}^{2} k_{x}^{2}+v_{y}^{2} k_{y}^{2}}$

Here,

$\lambda=+1(-1)$ is the band index which corresponds to conduction (valance) band and $k=\left(k_{x}, k_{y}\right)$ is the two dimensional momentum operator. Inclusion of triangular potential on the 8-Pmmn borophene can be written as,

$\widehat{H}=\widehat{H}_{\rho}+\widehat{H}_{\Delta}$

$\widehat{H}=\rho\left(v_{x} \hat{P}_{x} \sigma_{x}+v_{y} \hat{P}_{y} \sigma_{y}+v_{t} \hat{P}_{y} \sigma_{0}\right)+\beta_{0} \Delta_{t r i} \hat{P} \operatorname{Cos} 3 \theta$

$\widehat{H}_{\Delta}$ is triangular potential part of the borophene. $\beta_{0}$ is $\mathrm{I}_{2 \times 2}$ identity matrix and triangular potential velocity arises term is $\Delta_{t r i}$. The energy dispersion of the above $\widehat{H}$. Hamiltonian can be obtained by analytical method,

$E_{\rho}^{\lambda}=\frac{\Gamma 1\left(k_{x}, k_{y}\right)+\rho\left\lceil 2\left(k_{x}, k_{y}\right)+\lambda \mathrm{B}\left(k_{x}, k_{y}\right)\right.}{\left(k_{x}^{2}+k_{y}^{2}\right)^{2}}$

together with

$$
\begin{aligned}
& \Gamma 1\left(k_{x}, k_{y}\right)=k_{x}^{5}-2 k_{x}^{3} k_{y}^{2}-3 k_{x} k_{y}^{4} \\
& \Gamma 2\left(k_{x}, k_{y}\right)=k_{x}^{4} k_{y} \mathrm{v}_{\mathrm{t}}+2 k_{x}^{2} k_{y}^{3} \mathrm{v}_{\mathrm{t}}+k_{y}^{5} \mathrm{v}_{\mathrm{t}} \\
& \mathrm{B}\left(k_{x}, k_{y}\right)=\sqrt{\left(k_{x}^{2}+k_{y}^{2}\right)^{4}\left(v_{x}^{2} k_{x}^{2}+v_{y}^{2} k_{y}^{2}\right)}
\end{aligned}
$$

where $k_{i}$ is the momentum operators. $\Delta_{t r i}$ is an adjustable parameter which represents the triangular potential strength term. The adjustable parameter should be in the unit of velocity. Based on the calculations, we have chosen the strength of potential band parameter as a 1 .

Seen from the Figure 1 (a) that energy band dispersion in $k$-space $K$ valley of $8-P m m n$ borophene and Figure 1 (b) have also shown the top side of atomic structure of borophene. As known that, we can control the model with strength term but when we increase or decrease the term strength, the anisotropic triangular structure deteriorates, i.e., for the best anisotropic triangular spectrum, should be used the strength is 1 . We have confirmed this part with contour drawings of the energy spectrum with Figure 2 and the anisotropic circular spectrum appears to have evolved into an anisotropic triangle. Triangular potential strongly effects the anisotropic circular band structure of 2-dimensional borophene.

Even if the changes orientation of the triangular or circular band structure of the borophene, to adapt the problem in the $K$ or $K^{\prime}$ valley responds with the same intensity in both valleys. Additionally, anisotropic group velocity of the borophene arises due to tilted Dirac Fermions which depend on the energy eigenvalues of the system. In this study, we modify the energy eigenvalues of the system with triangular potential and the contribution of the triangular potential part on the

$$
\begin{aligned}
& v_{x}=\frac{1}{\hbar} \frac{\partial E_{\rho}^{\lambda}}{\partial k_{x}} \\
& v_{y}=\frac{1}{\hbar} \frac{\partial E_{\rho}^{\lambda}}{\partial k_{y}}
\end{aligned}
$$

energy eigenvalues of the bare borophene could not be neglected because its effect is really important on its energy. The relation of Dirac Fermion velocity and its relation to energy and momentum is obtained from,

where $E_{\rho}^{\lambda}$ is given in Eq.(4). Where $\rho$ represents to valley index, $+(-)$ corresponds to $K\left(K^{\prime}\right)$ Dirac points and $\lambda=1(-1)$ corresponds to electron (hole) states. Because of the anisotropy of the system along the

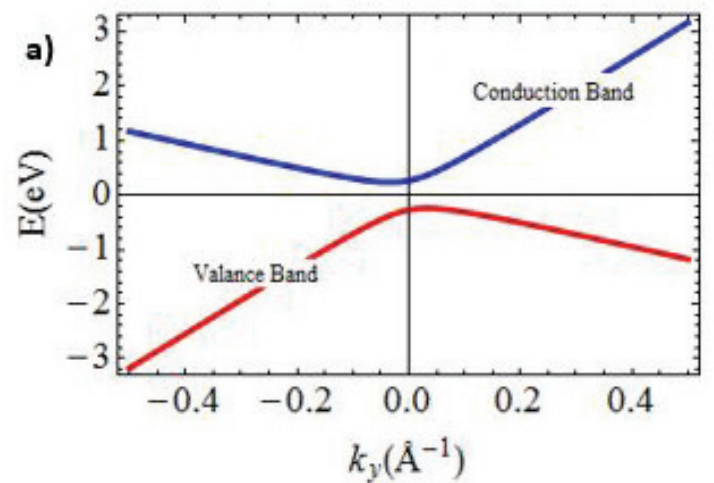

b)

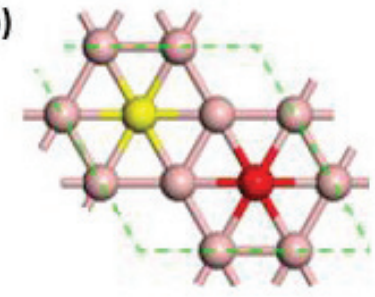

Figure 1. (a) Energy band dispersion in k-space representing equation (2) in valley K. (b) Top side view of atomic structure of borophene. The yellow and red balls denote the boron atoms move inward and outward from the plane, respectively [20]. 


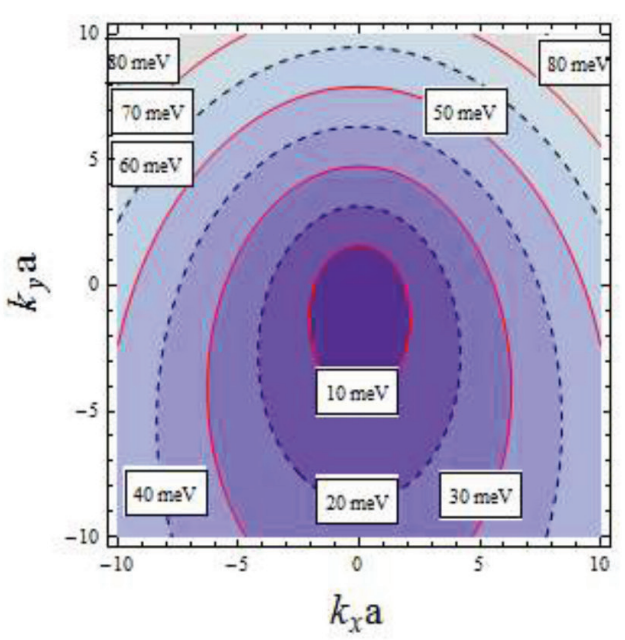

(a)

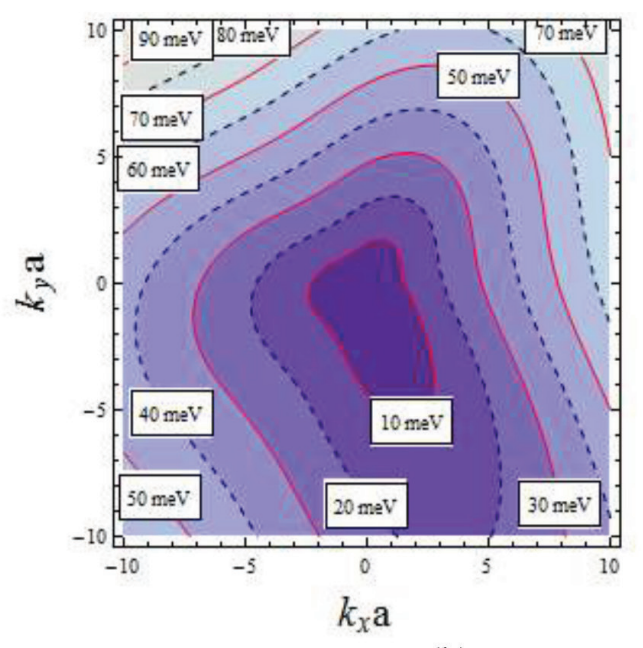

(b)

Figure 2. Fermi lines topology which are close to the $\mathrm{K}$ valley of the borophene a) circular anisotropic band structure $\mathrm{b}$ ) triangular anisotropic band structure.

axis-direction, electronic charge carriers have distinct from each other and have different Dirac Fermion velocity $\left\{v_{x}, v_{y}\right\}=\left\{0.86 v_{F}, 0.69 v_{F}\right\}$ By following the same procedure, the triangular potential dressed Dirac velocity can be found by expanding the Eq.(4) expression around to k-points. The renormalized fermi velocity of the effective charge carriers in 8-Pmmn borophene is available upon to evaluated in Figure 3 (Figure 4) with variable $k_{x}\left(k_{y}\right)$ values. In Figure $3 x$-direction effective Fermi velocity under the influence of triangular potential. Figure $3 a$ is corresponds to conduction band and Figure $3 \mathrm{~b}$ is respond to valance band of the effective energy. It is well-known that borophene

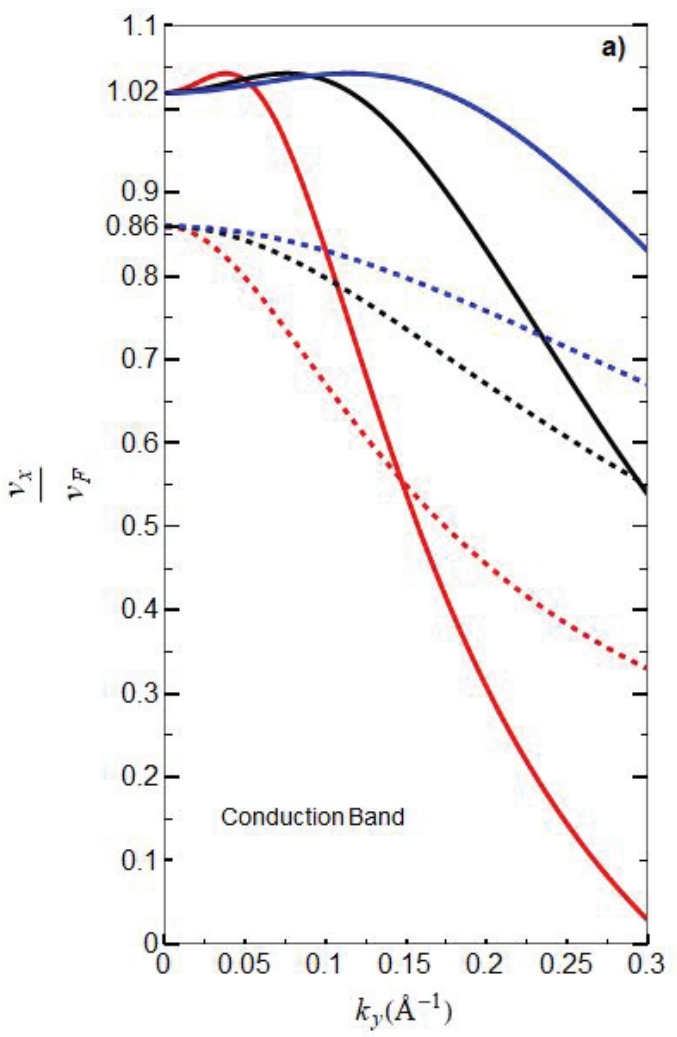

has different Fermion velocity $v_{x}$ is $0.86 v_{F}$. The value seen from the Figure $3 a$ (conduction band) and Figure $3 b$ (valance band) with dashed lines. \{red dashed lines, black dashed lines, blue dashed lines $\}=\left\{\left(0.1, k_{y}\right)\right.$, $\left.\left(0.2, k_{y}\right),\left(0.3, k_{y}\right)\right\}$. In conduction band $x$-direction effective Fermi velocity changes from $0.86 v_{F}$ to $1.02 v_{F}$ which represented with red lines, black lines, blue lines $\}=\left\{\left(0.1, k_{y}\right),\left(0.2, k_{y}\right),\left(0.3, k_{y}\right)\right\}$. The triangular potential dressed Fermi velocity $x$-direction in valance band can be found by following the same procedure which give changes from $0.86 v_{F}$ to $0.7 v_{F}$ in Figure $3 \mathrm{~b}$. One of the important implication that component momentum

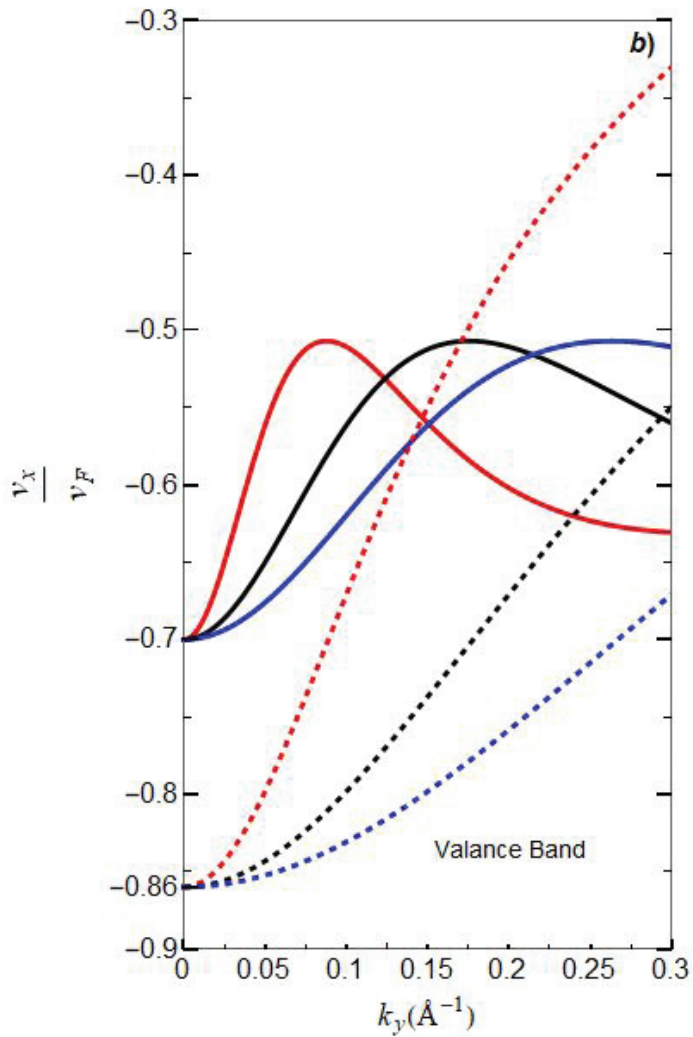

Figure 3. Renormalization $v_{x}$-direction of Fermi velocity in triangular potential dressed borophene with $k_{y}$ momentum. 

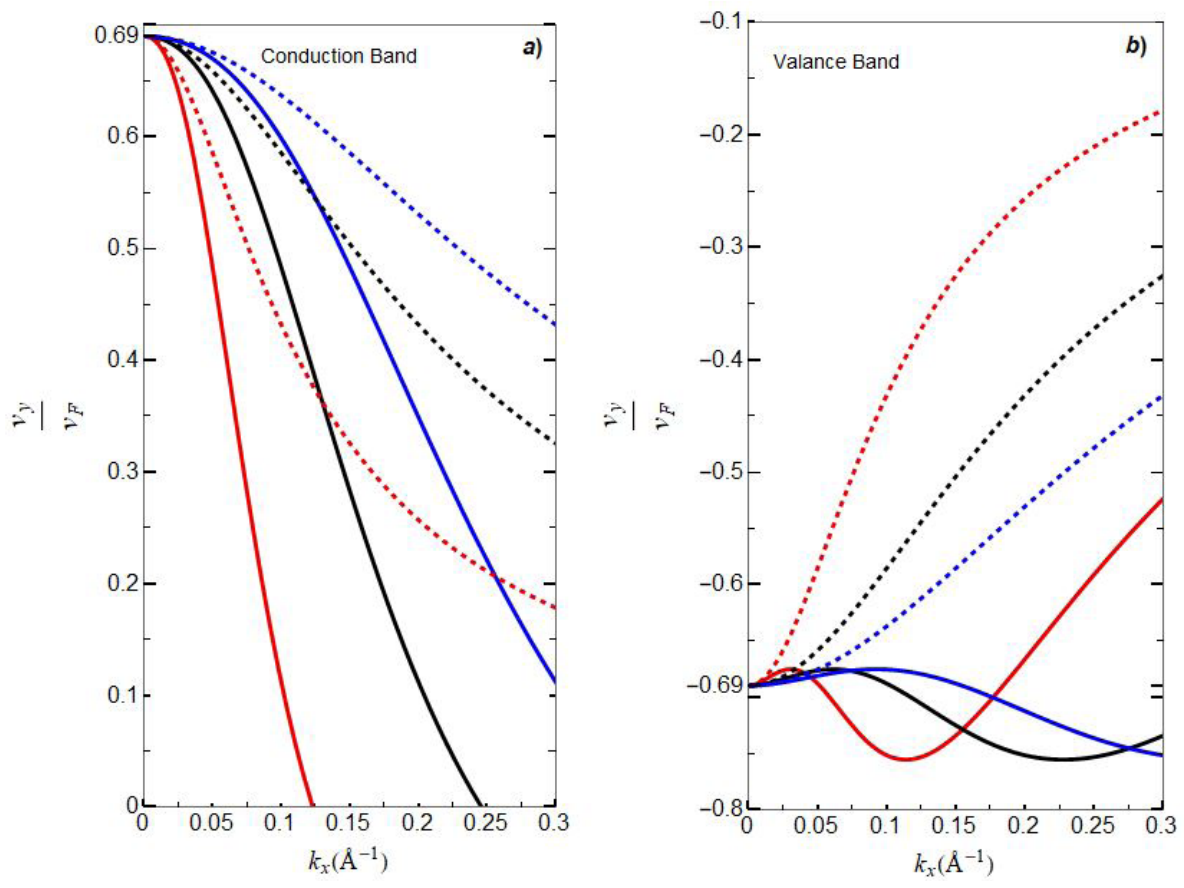

Figure 4. Renormalization $v_{y}$-direction of Fermi velocity in triangular potential dressed borophene with momentum.

responsible of the anisotropy so when dressed the Hamiltonian with any potential $k_{y}$ component is really effected. Additionally, we have to receive considerable critical attention that the behavior of renormalized velocity of x-component show different behaviour from the bare states.

With the same method, it is possible to investigate the renormalization $v_{y}$-direction of Fermi velocity in triangular potential dressed borophene with $k_{x}$ momentum. In Figure $4 \mathrm{a}$ and Figure $4 \mathrm{~b}$, we show the renormalization of the Fermi velocity along the $x$-direction which is quite different from the $y$-directions. In contrast to -direction of Fermi velocity in triangular potential dressed borophene, $v_{y}$-direction of Fermi velocity in triangular potential dressed borophene has not changed which has $0.69 v_{F}$. But, it is clearly seen from the figures that the behavior of the velocity has modified. That is, the $v_{x}$ and $v_{y}$ velocities along the $x$ and $y$ coordinates effected from the triangular potential and it is modify the intrinsic electronic properties of 8-Pmmn borophene.

\section{Conclusion}

This study aims to contribute to this growing area of research by exploring the electronic band spectrum of the 8-Pmmn borophene. The results in this study indicate that triangular potential is really effective parameter on the intrinsic electronic properties of 8-Pmmn borophene. The potential is an efficient band parameter which affecting the band properties of the system. We have used the theoretical analytic method to get the influence of triangular potential in the energy spectrum of the system. Following this technique, we have computed the anisotropic energy spectrum and the results show the same experimental setup of borophene [15]. After that, moves on to discuss in the presence of triangular potential. Due to the its highly anisotropic nature of the structure we have found that $x$-direction of the Fermi velocity decrease the value from $0.86 v_{F}$ to $0.7 v_{F}$ in valance band while $\mathrm{x}$-direction of the Fermi velocity increase the value from $0.86 v_{F}$ to $1.02 v_{F}$ in conduction band. But, even if $y$-direction of the Fermi velocity has not changed the value, triangular potential has changed the behavior of the Fermi velocity with respect to $k_{x}$ momentum. Although extensive research has been carried out on 8-Pmmn borophene, no single study exists which analyze triangular potential on the energy spectrum. The potential has non-negligible contribution to the electronic energy spectrum of borophene. As a conclusion, the results of conclusion can be extended with spin-orbit interactions which can be lead to better understanding for transport and electronic properties of the material.

\section{References}

[1] Novoselov K. S., Geim A. K., Morozov S. V., Jiang D., Zhang Y., Dubonos S. V., Grigorieva I. V., Firsov A. A., Electric field effect in atomically thin carbon films, Sci., 306, 666-669, 2004.

[2] Yao Y., Ye F., Qi X. L., Zhang S. C., Fang Z., Spin-orbit gap of graphene: Firstprinciples calculations, Phys. Rev. B 75, p. 041401(R), 2007.

[3] Boettger J. C. Trickey S. B., First-principles calculation of the spin-orbit splitting in graphene, Phys. Rev. B 75, p. 121402(R), 2007.

[4] Shakouri K., Masir M. R., Jellal A., Choubabi E., Peeters F., Effect of spin-orbit couplings in graphene with and without potential modulation, Phys. Rev. B 88, p. $115408,2013$. 
[5] Kandemir B. S., Akay D., The effect of electron-A1g phonon coupling in spin-orbit-coupled graphene, Philos. Mag 97, 2225, 2017.

[6] Akay D., Trigonal warping and photo-induced effects on zone boundary phonon in monolayer graphene, Superlattices Microstruct., 117, 18-24, 2018.

[7] Kandemir B. S., Akay D., Photoinduced Dynamical Band Gap in Graphene: The Effects of Electron-Phonon and Spin-Orbit Interaction, Phys. Status Solidi B , 255 (10), 2018.

[8] Auer C., Schürrer F., Ertler C., Hot phonon effects on the high-field transport in metallic carbon nanotubes, Phys. Rev. B, 74, 165409, 2006.

[9] Lazzeri M., Mauri F., Coupled dynamics of electrons and phonons in metallic nanotubes: Current saturation from hot-phonon generation, Phys. Rev. B 73, 165419, 2006.

[10] Xia F., Wang H., Xiao D., Dubey M., Ramasubramaniam A., Two-dimensional material Nanophotonics, Nat. Photonics, 8 (12), 899-907, 2014.

[11] Gupta A., Sakthivel T., Seal S. Recent development in 2D materials beyond graphene, Prog. Mater. Sci., 73, 44-126, 2015.

[12] Niu T., Li A. From two-dimensional materials to heterostructures, Prog. Surf. Sci. 90, 21-45, 2015.

[13] Mas-Ballesté R., Gómez-Navarro C., GómezHerrero J., Zamora F. 2D Materials: To graphene and beyond, Nanoscale, 3 (1), 20-30, 2011.

[14] Koppens F. H. L., Mueller T., Avouris P., Ferrari A. C., Vitiello M. S., Polini M., Photodetectors Based on Graphene, Other TwoDimensional Materials and Hybrid Systems. Nat. Nanotechnol. 9 (10), 780-793, 2014.

[15] Mannix A. J., Zhou X. F., Kiraly B., Wood J. D., Alducin D., Myers B. D., Liu X., Fisher B. L., Santiago U., Guest J. R., Yacaman M. J., Ponce A., Oganov A. R., Hersam M. C., Guisinger N. P., Synthesis of borophenes: Anisotropic, two-dimensional boron polymorphs., Sci, 350 (6267), 1513-1516, 2015.

[16] Verma S., Mawrie A., Ghosh T. K., Effect of electronhole asymmetry on optical conductivity in 8-Pmmn borophene, Phys. Rev., B 96, 155418, 2017.

[17] Lopez-Bezanilla A., Littlewood P. B., Electronic properties of 8-Pmmn borophene, Phys. Rev. B 93 241405, 2016.

[18] Li Z., Cao T., Wu M., Louie S. G., Generation of anisotropic massless dirac fermions and asymmetric klein tunneling in few-layer black phosphorus superlattices, Nano Lett. 17, 2280, 2017.

[19] Zabolotskiy A. D., Lozovik Y. E., Strain-induced pseudomagnetic field in the Dirac semimetal borophene, Phys. Rev. B 94 165403, 2016.

[20] [20] Xiao H., Cao W., Ouyang T., Guo S., He C., Zhong J., Lattice thermal conductivity of borophene from first principle calculation, Sci. Rep. 7, 45986, 2017. 